\title{
HARMONIC AND RELATIVE HARMONIC DIMENSIONS
}

\author{
MITSURU NAKAI and LEO SARIO
}

Consider an open Riemann surface $R$ of Heins type, i.e., a parabolic Riemann surface with a single ideal boundary component $\delta R$. Let $K$ be a closed parametric disk on $R$ and $H P(R-K ; \partial(R-K))$ the class of nonnegative harmonic functions on $R-K$ with vanishing boundary values on the relative boundary $\partial(R-K)$ of $R-K$. The cardinal number of the set of nonproportional minimal functions in $H P(R-K ; \partial(R-K))$, is, by definition, the harmonic dimension $\operatorname{dim} \delta R$ of the ideal boundary $\delta R$ of $R$. It is at least 1 and independent of the choice of $K$. The notion of harmonic dimension of the ideal boundary of a surface of what we shall call Heins type was introduced by Heins in [4].

Let $F$ be the union of a locally finite family of disjoint closed parametric disks on $R$ and define $H P(R-F ; \partial(R-F))$ as above. The cardinal number of the set of nonproportional minimal functions in $H P(R-F ; \partial(R-F))$ is known as the relative harmonic dimension $\operatorname{dim}_{F} \delta R$ of the ideal boundary $\delta R$ of $R$ relative to $F$. Again it is at least 1, but this time it depends essentially on $F$ unless $F$ is compact, in which case $\operatorname{dim}_{F} \delta R=\operatorname{dim} \delta R$. A more detailed description of these concepts will be given in Section 1 .

The first purpose of this paper is to compare $\operatorname{dim} \delta R$ and $\operatorname{dim}_{F} \delta R$. We shall see that all three cases can occur, depending on the choice of $R$ and $F: \operatorname{dim}_{F} \delta R<$ $\operatorname{din} \delta R, \operatorname{dim}_{F} \delta R>\operatorname{dim} \delta R$, and $\operatorname{dim}_{F} \delta R=\operatorname{dim} \delta R$. In Section 2 we shall show that, on any $R$, there exists an $F$ such that $\operatorname{dim}_{F} \delta R=1$; therefore, $\operatorname{dim}_{F} \delta R<\operatorname{dim} \delta R$ if the latter exceeds 1 . In Section 3 an example will be given of an $F$ in the complex plane $\boldsymbol{C}$ with $\operatorname{dim}_{F} \delta \boldsymbol{C}>1$; since $\operatorname{dim} \delta \boldsymbol{C}=1$, we have an $R$ and an $F$ with $\operatorname{dim}_{F} \delta R>\operatorname{dim} \delta R$. The most important task in this context is to characterize those $F$ for which $\operatorname{dim}_{F} \delta R=\operatorname{dim} \delta R$. We shall give in Section 4 a useful sufficient condition.

The second purpose of this paper is to construct an example of a Riemann surface $R$ of Heins type with $\operatorname{dim} \delta R=\mathfrak{a}$, the cardinal number of a countably infinite set. This will be done in Section 5, our method demonstrating the applicability of the sufficient conditions obtained in Section 4. An example of $\operatorname{dim} \delta R=\mathfrak{a}$ was first given by Kuramochi [5], and quite recently by Segawa [11], who used his duality theorem. It was Heins [4] who gave an example of a Riemann surface $R$ of Heins type with $\operatorname{dim} \delta R=n$, an arbitrary positive integer. If one tries to follow the Heins 
construction for the case $\operatorname{dim} \delta R=\mathfrak{a}$ as well, he is led in a natural manner to the concept of relative harmonic dimension. Anybody who visualizes this is convinced of the fact that the example of $R$ with $\operatorname{dim} \delta R=\mathfrak{a}$ already existed implicitly in the pioneering work of Heins. This observation is a motivation of our present study of the relative harmonic dimension.

\section{Relative harmonic dimension}

1.1. We denote by $\delta R$ the ideal boundary of an open Riemann surface $R$. By this we mean that $\delta R$ is an abstract set and $R \cup \delta R$ is topologized as a compact Hausdorff space containing $R$ as its open and dense subset. Unless otherwise explicitly stated, we do not specify the topology of $R \cup \delta R$ beyond this requirement. However, we often wri e $\zeta \rightarrow \delta R(\zeta \in R)$ to mean that $\zeta$ converges to the point at infinity of $R$ with $R \cup \delta R$ then understood as the Alexandroff compactification of $R$.

We say that $R$ has a single ideal boundary component $\delta R$ if $\delta R$ is connected when $R \cup \delta R$ is realized as the Kerékjártó-Stoïlow compactification (cf., e.g., [3]). We shall say that a Riemann surface is of Heins type if it is of the kind first systematically studied by Heins [4]: an open surface of parabolic type with a single ideal boundary component $\delta R$. The complex plane $\boldsymbol{C}$ and the punctured sphere $\hat{\boldsymbol{C}}_{0}=\hat{\boldsymbol{C}}-\{0\}$ are typically of Heins type.

If $R$ is of Heins type, then there exists an exhaustion $\left\{R_{n}\right\}_{0}^{\infty}$ of $R$ with the following properties: i) each $R_{n}$ is a relatively compact region and $\partial R_{n}$ is an analytic Jordan curve, ii) $R_{n} \subset \bar{R}_{n} \subset R_{n+1} \quad(n=0,1,2, \ldots)$, iii) $\bigcup_{n=0}^{\infty} R_{n}=R$, iv) the functions $w_{n} \in C(R) \cap H\left(R_{n}-\bar{R}_{0}\right)$ with $w_{n} \mid \bar{R}_{0}=0$ and $w_{n} \mid R-R_{n}=1$ satisfy $\lim _{n \rightarrow \infty} w_{n}=0$, uniformly on each compact subset of $R$. Conversely, if $R$ has an exhaustion with properties i)-iv), then $R$ is of Heins type. Here $H(S)$ is the class of harmonic functions on a Riemann surface $S$. We also denote by $H P(S)$ the class of nonnegative functions in $H(S)$. A function $u$ in $H P(S)$ is said to be minimal in $H P(S)$ if $u>0$ and $u \geqq v \geqq 0$ for any $v$ in $H P(S)$ implies that $v / u$ is constant on $S$.

1.2. Let $S$ be a subregion of an open Riemann surface $R$ such that each point in $\partial S$ is regular for the Dirichlet problem for the region $S$. We consider the relative class

$$
H P(S ; \partial S)=\{u \in C(R) \cap H P(S) ; u \mid R-S=0\} .
$$

A function $u$ in $H P(S ; \partial S)$ is, by definition, minimal in $H P(S ; \partial S)$ if $u$ is not identically zero and $u \geqq v \geqq 0$ for any $v$ in $H P(S ; \partial S)$ implies that $v / u$ is constant on $S$, i.e., there exists a constant $c$ such that $v=c u$ on $R$. Clearly, if $u$ is minimal in $H P(S ; \partial S)$, then $u$ is minimal in $H P(S)$, but not conversely.

With class (1), we associate two mappings, $\lambda$ and $\mu$, defined as follows. For each $u$ in $H P(R)$, let $\lambda u$ be the upper envelope of the family of functions $v$ in $H P(S ; \partial S)$ 
with $v<u$. Then $\lambda$ is a homogeneous, additive, and order preserving mapping of $H P(R)$ to $H P(S ; \partial S)$. We denote by $H P(S ; \partial S)_{\mu}$ the class of functions $v$ in $H P(S ; \partial S)$ with harmonic majorants on $R$. For each $v$ in $H P(S ; \partial S)_{\mu}$, let $\mu v$ be the least harmonic majorant of $v$ on $R$. Then $\mu$ is a homogeneous, additive, and order preserving mapping of $H P(S ; \partial S)_{\mu}$ to $H P(R)$. We will use the following properties of $\lambda$ and $\mu$ (cf., e.g., Noshiro [10], pp. 102-103):

(a) $\lambda \mu v=v$ for every $v$ in $H P(S ; \partial S)_{\mu}$ so that $\mu$ is injective, and $\lambda$ is injective on $\mu\left(H P(S ; \partial S)_{\mu}\right)$.

(b) If $u \in H P(R)$ satisfies $u \leqq \mu v$ for some $v$ in $H P(S ; \partial S)_{\mu}$, then $u$ belongs to $\mu\left(H P(S ; \partial S)_{\mu}\right)$.

(c) $v \in H P(S ; \partial S)_{\mu}$ is minimal in $H P(S ; \partial S)$ if and only if $\mu v$ is minimal in $H P(R)$.

(d) If $u$ is minimal in $H P(R)$ and $\lambda u>0$ on $S$, then $\lambda u$ is minimal in $H P(S ; \partial S)$.

1.3. Unless otherwise explicitly stated, we consider henceforth exclusively open Riemann surfaces $R$ of Heins type. Take a finite or countably infinite sequence $\left\{K_{n}\right\}=\left\{K_{n}\right\}_{1}^{N}(1 \leqq N \leqq \infty)$ of nondegenerate compact continua $K_{n}$ in $R$ with the following conditions: $\left.\alpha) K_{n} \cap K_{m}=\emptyset(n \neq m), \beta\right)\left\{K_{n}\right\}$ is locally finite, i.e., the set $\left\{n ; K_{n} \cap X \neq \emptyset\right\}$ is finite for any compact subset $X$ of $\left.R, \gamma\right) R-\bigcup_{1}^{N} K_{n}$ is connected. Such a sequence $\left\{K_{n}\right\}$ will be called a $\mathscr{K}$-sequence in this paper. With a $\mathscr{K}$-sequence $\left\{K_{n}\right\}$ we associate a closed set $F$ and a region $W$ given by

$$
F=\bigcup_{1}^{N} K_{n}, \quad W=R-F
$$

We also fix a reference point $a$ in $W$ and denote by $M(W)$ the class of minimal functions $v$ in $H P(W ; \partial W)$ with $v(a)=1$. We call the cardinal number $\# M(W)$ of $M(W)$ the relative harmonic dimension of $\delta R$ with respect to $F$ and denote it by $\operatorname{dim}_{F} \delta R$ :

$$
\operatorname{dim}_{F} \delta R=\# M(W)
$$

Clearly it is independent of the choice of the reference point $a$.

Let $\left\{K_{i n}\right\}_{n=1}^{N_{i}}(i=1,2)$ be two $\mathscr{K}$-sequences on $R$ and set $F_{i}=\bigcup_{1}^{N_{i}} K_{i n}$ and $W_{i}=R-F_{i}(i=1,2)$. Obviously $F_{i}$ is compact on $R$ if and only if $N_{i}<\infty$. Suppose $F_{1}$ and $F_{2}$ are compact on $R$. Then it is not difficult to prove that there exists a bijective, homogeneous, additive, and order preserving mapping $u_{1} \mapsto u_{2}$ of $H P\left(W_{1} ; \partial W_{1}\right)$ to $H P\left(W_{2} ; \partial W_{2}\right)$ such that $u_{1}-u_{2}$ is bounded near $\delta R$. Hence \# $M\left(W_{1}\right)=\# M\left(W_{2}\right)$ and a fortiori $\operatorname{dim}_{F_{1}} \delta R=\operatorname{dim}_{F_{2}} \delta R$. The quantity

$$
\operatorname{dim} \delta R=\operatorname{dim}_{F} \delta R \quad(F \text { compact })
$$

is thus uniquely determined, with a compact $F$ chosen at will. This quantity, an appropriate one attached to $R$, is called the harmonic dimension of $\delta R$.

1.4. Let $\left\{K_{n}\right\}$ be an arbitrary $\mathscr{K}$-sequence in $R$ and let $F$ and $W$ be associated with $\left\{K_{n}\right\}$ as in (2). Denote by $W^{*}$ the Martin compactification of $W$ and by $k_{W}(z, \zeta)$ 
the Martin kernel on $W^{*}$ with the reference point $a$ in $W$ (cf., e. g., [3]):

$$
k_{W}(z, \zeta)=\frac{g_{W}(z, \zeta)}{g_{W}(a, \zeta)}
$$

for $(z, \zeta)$ in $W \times W$, with $g_{W}(z, \zeta)$ the Green's function on $W$. For any $q$ in $W^{*}-W$ there exists a sequence $\left\{\zeta_{n}\right\}$ in $W$ such that $\zeta_{n} \rightarrow q$ in $W^{*}$ and either $\zeta_{n} \rightarrow \delta R$ or any subsequence of $\left\{\zeta_{n}\right\}$ contains a subsequence converging to a point of $F$. We denote by $Q(W)$ the class of the points $q$ in $W^{*}-W$ over $\delta R$, i.e., those $q$ in $W^{*}-W$ for which the first alternative occurs. One can easily see that $Q(W)$ is compact in $W^{*}$. We also denote by $Q_{1}(W)$ the class of minimal points $q$ over $\delta R$, i.e., those points $q$ in $Q(W)$ for which $k_{W}(\cdot, q) \in M(W)$. By the Martin theory (cf., e.g., [3], pp. 134-144),

$$
M(W)=\left\{k_{W}(\cdot, q) ; \quad q \in Q_{1}(W)\right\},
$$

and there exists a bijective correspondence $u \leftrightarrow v$ between $H P(W ; \partial W)$ and the class of positive Borel measures $v$ on $Q_{1}(W)$ such that

$$
u=\int_{Q_{1}(W)} k_{W}(\cdot, q) d v(q) .
$$

As a consequence of (6) we have $\operatorname{dim}_{F} \delta R \geqq 1$ and $\operatorname{dim} \delta R \geqq 1$. Needless to say, $\operatorname{dim}_{F} \delta R$ and $\operatorname{dim} \delta R$ are at most $\mathfrak{c}$, the cardinal number of a continuum.

\section{The smallest relative harmonic dimension}

2.1. By a closed Jordan region on a Riemann surface we mean the closure of a Jordan region on it. One might feel that $\operatorname{dim}_{F} \delta R$ for compact $F$, i.e., $\operatorname{dim} \delta R$, never exceeds $\operatorname{dim}_{F} \delta R$ for any noncompact $F$. Contrary to this intuition we have the following

Theorem. For any open Riemann surface $R$ of Heins type, there always exists a $\mathscr{K}$-sequence $\left\{K_{n}\right\}_{1}^{\infty}$ of closed Jordan regions $K_{n}$ on $R$ such that $\operatorname{dim}_{F} \delta R=1$ for $F=\bigcup_{1}^{\infty} K_{n}$.

The proof will be given in $2.2-2.4$. What we need to show is that $H P(W ; \partial W)$ is generated by a single nonzero element $k$ in $H P(W ; \partial W)$, i.e., $H P(W ; \partial W)=$ $\left\{\alpha k ; \alpha \in \boldsymbol{R}^{+}\right\}$, where $\boldsymbol{R}^{+}$is the set of nonnegative numbers in the set $\boldsymbol{R}$ of real numbers. It will be seen from the proof that we do not use the parabolicity of $R$. Therefore, what we can really assert is the following: For any open Riemann surface $R$ with a single ideal boundary component $\delta R$, there exists a sequence $\left\{K_{n}\right\}_{1}^{\infty}$ of disjoint closed Jordan regions $K_{n}$ converging to $\delta R$ such that $H P(W ; \delta W)$ is generated by a single nonzero element.

2.2. Since $R$ has a single ideal boundary component, there exists an exhaustion $\left\{R_{n}\right\}_{0}^{\infty}$ of $R$ with properties i), ii), and iii) stated in 1.1 . We may choose $R_{0}$ as a para- 
metric disk. For each $n \geqq 0$, take a regular subregion $S_{n}$ of $R$ such that $\bar{R}_{n} \subset S_{n} \subset$ $\bar{S}_{n} \subset R_{n+1}$ and $S_{n}-\bar{R}_{n}$ is an annulus. For $n \geqq 1$, let $\varphi_{n}$ be a conformal mapping of $S_{n}-\bar{R}_{n}$ onto the region $\left\{1<|t|<a_{n}\right\}$ such that $\partial S_{n}$ and $\partial R_{n}$ correspond to the circles $\{|t|=1\}$ and $\left\{|t|=a_{n}\right\}$, respectively, under the mapping $\varphi_{n}$ extended to $\bar{S}_{n}-R_{n}$. For a number $\delta_{n}$ in $(0, \pi)$ to be specified later, the set

$$
K_{n}=\varphi_{n}^{-1}\left\{1 \leqq|t| \leqq a_{n}, \delta_{n} \leqq \arg t \leqq 2 \pi-\delta_{n}\right\} \quad(n=1,2, \ldots)
$$

is a closed Jordan region on $R$. Clearly, $K_{n} \cap K_{m}=\emptyset(n \neq m)$, and $\left\{K_{n}\right\}$ converges to $\delta R$. For convenience we include $K_{0}=\bar{R}_{0}$ in $\left\{K_{n}\right\}$ and set

$$
F=\bigcup_{0}^{\infty} K_{n}, \quad W=R-F
$$

We shall prove that $H P(W ; \partial W)$ is generated by a single nonzero element if $\left\{\delta_{n}\right\}$ is properly chosen in $(0, \pi)$.

2.3. For $n \geqq 1$ we consider the arc $I_{n}=\varphi_{n}^{-1}\left\{|t|=a_{n},-\delta_{n} \leqq \arg t \leqq \delta_{n}\right\}$ on $\partial R_{n}$ and fix a point $\zeta_{n}$ in $I_{n}$ with $\varphi_{n}\left(\zeta_{n}\right)=a_{n}$ so that $\zeta_{n}$ is the midpoint of $I_{n}$. We denote by $g_{n}(\zeta, z)$ the Green's function on $R_{n} \cap W$. We use the same symbol $\zeta$ for a point of $R$ and its image in a parametric disk. The inner normal derivative $\partial / \partial n_{\zeta} g_{n}(\zeta, z)$ of $g_{n}(\zeta, z)$ at $\zeta$ on $\partial R_{n}$ depends on the choice of the parametric disk but, for a fixed point $a$ in $R_{1} \cap W$, the ratio

$$
h_{n}(\zeta, z)=\left(\frac{\partial}{\partial n_{\zeta}} g_{n}(\zeta, z)\right) /\left(\frac{\partial}{\partial n_{\zeta}} g_{n}(\zeta, a)\right)
$$

for $(\zeta, z)$ in $\left(\partial R_{n}\right) \times\left(R_{n} \cap W\right)$ does not. Since $h_{n}(\zeta, z)$ is continuous on $\left(\partial R_{n}\right) \times$ $\left(R_{n} \cap W\right)$, the function $\zeta \rightarrow h_{n}(\zeta, z)$ is uniformly continuous on $\partial R_{n}$ for each $z$ in $\partial S_{0} \subset R_{n} \cap W$. Hence the function

$$
\psi_{n}(\zeta)=\sup _{z \in \partial S_{0}}\left|h_{n}(\zeta, z)-h_{n}\left(\zeta_{n}, z\right)\right|
$$

is nonnegative and continuous on $\partial R_{n}$, with $\psi_{n}\left(\zeta_{n}\right)=0$. Here $\psi_{n}$ depends on $\delta_{1}, \ldots$, $\delta_{n-1}$ but does not depend on $\delta_{n}$. If we take $\delta_{n}$ sufficiently small in $(0, \pi)$, then $\sup _{\zeta \in I_{n}} \psi_{n}(\zeta)<2^{-n}$. Therefore we can and will choose $\delta_{n}(n=1,2, \ldots)$ successively in $(0, \pi)$ so small that

$$
\sup _{\zeta \in I_{n}}\left(\sup _{z \in \partial S_{0}}\left|h_{n}(\zeta, z)-h_{n}\left(\zeta_{n}, z\right)\right|\right)<2^{-n} \quad(n=1,2, \ldots) .
$$

2.4. We now determine the generator $k$ of $H P(W ; \partial W)$. To this end we consider functions $k_{n}(z)=h_{n}\left(\zeta_{n}, z\right)(n=1,2, \ldots)$ which are in $H P\left(R_{n} \cap W ; \partial\left(R_{n} \cap W\right)-\left\{\zeta_{n}\right\}\right)$, the class of nonnegative harmonic functions on $R_{n} \cap W$ with vanishing boundary values on $\partial\left(R_{n} \cap W\right)-\left\{\zeta_{n}\right\}$. We recall that $k_{n}(a)=1$. Since $\left\{k_{n}\right\}_{1}^{\infty}$ forms a normal family, there exists a subsequence $\left\{k_{v(n)}\right\}_{n=1}^{\infty}$ of $\left\{k_{n}\right\}_{1}^{\infty}$ such that

$$
k(z)=\lim _{n \rightarrow \infty} k_{v(n)}(z)
$$


exists on $\bar{W}$ and the convergence is uniform on each compact subset of $\bar{W}$. Clearly $k \in H P(W ; \partial W)$ and $k(a)=1$.

Choose an arbitrary element $v$ in $H P(W ; \partial W)$ with $v(a)=1$. The proof will be complete if we can show that $v=k$. Since $\partial\left(R_{n} \cap W\right)$ is piecewise analytic, $* d g_{n}(\cdot, z)$ exists on $\partial\left(R_{n} \cap W\right)$ except at corner points. But since $v$ vanishes on those components of $\partial\left(R_{n} \cap W\right)$ which contain these corner points, the Poisson-type formula is valid:

$$
v(z)=-\frac{1}{2 \pi} \int_{\partial\left(R_{n} \cap W\right)} v * d g_{n}(\cdot, z) \quad\left(z \in R_{n} \cap W\right) .
$$

Observe that the boundary function $v \mid \partial\left(R_{n} \cap W\right)$ is nonvanishing on $I_{n}\left(\subset \partial R_{n}\right)$. Therefore we can rewrite the above formula as

$$
v(z)=\int_{I_{n}} \frac{1}{2 \pi}\left(\frac{\partial}{\partial n_{\zeta}} g_{n}(\zeta, z)\right) v(\zeta)|d \zeta| \quad\left(z \in R_{n} \cap W\right) .
$$

Using a positive measure $\mu_{n}$ on $I_{n}$ defined by

$$
d \mu_{n}(\zeta)=\frac{1}{2 \pi} \frac{\partial}{\partial n_{\zeta}}\left(g_{n}(\zeta, a)\right) v(\zeta)|d \zeta|,
$$

and recalling the definition of $h_{n}(\zeta, z)$ we obtain

$$
v(z)=\int_{I_{n}} h_{n}(\zeta, z) d \mu_{n}(\zeta) \quad\left(z \in R_{n} \cap W\right) .
$$

For $z=a$ this, implies that $\mu_{n}\left(I_{n}\right)=1$. Set $\Omega=S_{0}-\bar{R}_{0}$. If $z \in \Omega$, then (8) gives

$$
\begin{gathered}
\left|v(z)-k_{v(n)}(z)\right|=\left|\int_{I_{v(n)}}\left(h_{v(n)}(\zeta, z)-h_{v(n)}\left(\zeta_{v(n)}, z\right)\right) d \mu_{v(n)}(\zeta)\right| \\
\leqq \int_{I_{v(n)}}\left|h_{v(n)}(\zeta, z)-h_{v(n)}\left(\zeta_{v(n)}, z\right)\right| d \mu_{v(n)}(\zeta)<2^{-v(n)} .
\end{gathered}
$$

We have shown that $\sup _{z \in \bar{\Omega}}\left|v(z)-k_{v(n)}(z)\right| \leqq 2^{-v(n)}$. On letting $n \rightarrow \infty$ and using (9) we see that $v=k$ on $\Omega$ and hence on $W$.

2.5. Examples of Riemann surfaces $R$ of Heins type with $\operatorname{dim} \delta R>1$ are not lacking. As already mentioned in the introduction, Heins [4] constructed an $R$ with $\operatorname{dim} \delta R=n$, an arbitrary positive integer, and Kuramochi [5] exhibited an $R$ with $\operatorname{dim} \delta R=\mathfrak{a} \quad(=\# \boldsymbol{N}$, with $N$ the set of positive integers) (see also Segawa [11]). Constantinescu and Cornea [2] even constructed an $R$ with $\operatorname{dim} \delta R=\mathfrak{c}(=\# \boldsymbol{R})$. We shall also construct, in Section 5, an $R$ with $\operatorname{dim} \delta R=\mathfrak{a}$. From Theorem 2.1 we thus conclude that there exists an open Riemann surface $R$ of Heins type and a $\mathscr{K}$-sequence $\left\{K_{n}\right\}_{1}^{\infty}$ on $R$ such that for $F=\cup_{1}^{\infty} K_{n}$,

$\operatorname{dim}_{F} \delta R<\operatorname{dim} \delta R$. 


\section{The finite complex plane}

3.1. The classical Picard principle states that the harmonic dimension of the point at infinity, $\infty=\delta C$, of the finite complex plane $C:|z|<\infty$ is one $: \operatorname{dim} \delta C=1$. Let $\left\{K_{n}\right\}_{1}^{\infty}$ be a $\mathscr{K}$-sequence of radial slits $K_{n}$ on $C$ and $F=\cup_{1}^{\infty} K_{n}$. We can always find an $F$ with $\operatorname{dim}_{F} \delta C=m$ for any cardinal number $m \geqq 1$ of a countable set or the cardinal number $m$ of a continuum (cf., e.g., [6], [7], [9]). Therefore, the relation $\operatorname{dim}_{F} \delta R \geqq \operatorname{dim} \delta R$ occurs frequently. For the sake of completeness we append here an example of extreme simplicity (both in the example itself and its proof) of a $\mathscr{K}$ sequence $\left\{K_{n}\right\}_{1}^{\infty}$ in $C$ such that $\operatorname{dim}_{F} C \geqq 2$ for $F=\bigcup_{1}^{\infty} K_{n}$; our example was inspired by Ancona [1]. In particular, we have a proof of the occurrence of the relation

$$
\operatorname{dim}_{F} \partial R>\operatorname{dim} \partial R \text {. }
$$

3.2. Consider a nondegenerate continuum $K$ in $C$ with the following four properties: (K.1) $K$ is symmetric about the real axis $\operatorname{Im} z=0$, (K.2) $K$ is symmetric about the imaginary axis $\operatorname{Re} z=0$, (K.3) $(K+1) \cap K=\emptyset$, (K.4) $C-K$ is connected. Here $K+c=\{z+c ; z \in K\}$ for any given $c \in C$. The slit $[-a, a](0<a<1 / 2)$, the disk $\{|z| \leqq a\} \quad(0<a<1 / 2)$, and the rectangle $\{|\operatorname{Re} z| \leqq a,|\operatorname{Im} z| \leqq b\} \quad(0<a<1 / 2)$, $(b>0)$ are examples of $K$. Let $Z=\{0, \pm 1, \pm 2, \ldots\}$ and

$$
K_{n}=K+n \quad(n \in Z), \quad F=\bigcup_{-\infty}^{\infty} K_{n} .
$$

Theorem. Let $K$ be a nondegenerate continuum $K$ in $C$ with properties (K.1)-(K.4), and let $F$ be given by (10). Then $\operatorname{dim}_{F} \delta C \geqq 2$.

It can be seen that, in reality, $\operatorname{dim}_{F} \delta C=2$ but our main interest here is in constructing an $F$ with $\operatorname{dim}_{F} \delta R>\operatorname{dim} \delta R$ and also in giving a proof as simple and elementary as possible. Hence we only establish $\operatorname{dim}_{F} \delta C \geqq 2$ and omit the proof for $\operatorname{dim}_{F} \delta C \leqq 2$, which requires rather elaborate reasoning.

3.3. Our proof of Theorem 3.2 is by contradiction. Suppose $\operatorname{dim}_{F} \delta C=1$, so that there exists a nonzero function $u$ in $H P(W ; \partial W)$ with $W=C-F$ such that $H P(W ; \partial W)=\boldsymbol{R}^{+} u$. Since $v$ defined by $v(z)=2^{-1}(u(z)+u(\bar{z}))$ for $z$ in $W$ is also a function in $H P(W ; \partial W)$ by (K.1), we may assume that $u(\bar{z})=u(z)$ for $z$ in $W$. Similarly, the function $v$ given by $v(z)=2^{-1}(u(z)+u(-\bar{z}))$ for $z$ in $W$ is again in $H P(W ; \partial W)$ by (K.2). Hence we may and will assume that $u(z)=u(\bar{z})=u(-\bar{z})$ for any $z$ in $W$.

Let $I_{n}$ be the line segment contained in $\{\operatorname{Im} z=0\} \cap \bar{W}$ connecting the rightmost point of $\{\operatorname{Im} z=0\} \cap K_{n}$ to the leftmost point of $\{\operatorname{Im} z=0\} \cap K_{n+1}(n \in Z)$. We maintain that

$$
\left(u \mid I_{k}\right)(z)=\left(u \mid I_{k+1}\right)(2(k+1)-z)
$$

for $z \in I_{k}$, i.e., $u \mid I_{k}$ and $u \mid I_{k+1}$ are symmetric about $\{\operatorname{Re} z=k+1\}$. In fact, let 
$v \in H P(W ; \partial W)$ be given by $v(z)=u(z+(k+1))$ for $z$ in $W$. Then (11) for $k$ follows from $v(z)=v(-\bar{z})$ for $z$ in $W$. Since $v \in H P(W ; \partial W)=\boldsymbol{R}^{+} u$, there exists a constant $c$ in $\boldsymbol{R}^{+}$with $v=c u$. Therefore, $u(z)=u(-\bar{z})$ implies $v(z)=v(-\bar{z})$ for any $z$ in $W$.

Let $W^{+}=W \cap\{\operatorname{Im} z>0\}$. From (11) it follows that $u \mid \partial W^{+}$is bounded and a fortiori the solution $H_{u}^{W^{+}}$of the Dirichlet problem on $W^{+}$with boundary values $u \mid \partial W^{+}$on $\partial W^{+}$(cf., e.g., [3], p. 21) is bounded on $W^{+}$. Note that $H_{u}^{W^{+}}$ is continuous on $\overline{W^{+}}$with $H_{u}^{W^{+}}\left|\partial W^{+}=u\right| \partial W^{+}$. Define the harmonic function $h$ on $W$ by

$$
h(z)=\left\{\begin{array}{cl}
u(z)-H_{u}^{W^{+}}(z) & \left(z \in \overline{W^{+}}\right), \\
-\left(u(\bar{z})-H_{u}^{W^{+}}(\bar{z})\right) & \left(z \in W-\overline{W^{+}}\right) .
\end{array}\right.
$$

Since $u(z)=u(\bar{z})$, the function $w$ defined by $w=u+h$ belongs to $H P(W ; \partial W)$ and is unbounded (bounded, respectively) on $\overline{W^{+}}\left(W-\overline{W^{+}}\right.$, respectively). Thus $u$ is not symmetric about the real axis, in violation of the fact that $w$ is a constant multiple of $u$. In the above proof we took it for granted that $u$ is unbounded on $W$. If this were not the case, the parabolic character of $\delta C$ would imply $u \equiv 0$.

\section{Identity}

4.1. Take a $\mathscr{K}$-sequence $\left\{K_{n}\right\}_{1}^{N}$ on $R$ and set $F=\bigcup_{1}^{N} K_{n}$ and $W=R-F$. We now take up the most intriguing case: when is $\operatorname{dim}_{F} \delta R=\operatorname{dim} \delta R$ ? If $F$ is compact, the identity holds by definition. This suggests that, for a noncompact $F$, the identity occurs whenever $\left\{K_{n}\right\}$ is distributed on $R$ sparsely in some sense. We shall give here a condition to assure such sparseness. Let $g_{W}(z, \zeta)$ be the Green's function on $W$. A curve $\gamma$ in $R$ is said to converge to $\delta R, \gamma \rightarrow \delta R$, if $\lim _{t \rightarrow 1} p(t)=\delta R$, with $p=p(t)$ $(0 \leqq t<1)$ a parametric representation of $\gamma$. The curvewise superior limit of $g_{W}(z, \zeta)$ along $\gamma$ is, by definition,

$$
\limsup _{z \in \gamma, z \rightarrow \delta R} g_{W}(z, \zeta)=\limsup _{t \rightarrow 1} g_{W}(p(t), \zeta),
$$

where we define $g_{W}(\cdot, \zeta)=0$ on $R-W=F$. We say that $\left\{K_{n}\right\}$ is sparse on $R$ if the curvewise superior limit of $g_{W}(\cdot, \zeta)$ along any curve $\gamma$ in $R$ converging to $\delta R$ is positive. The condition is clearly independent of $\zeta$ in $W$. If $F$ is compact, then, since $R$ is parabolic, we have

$$
\liminf _{z \rightarrow \delta R} g_{W}(z, \zeta)>0
$$

and therefore $\left\{K_{n}\right\}_{1}^{N}\left(F=\bigcup_{1}^{N} K_{n}\right)$ is sparse on $R$.

Theorem. If $\left\{K_{n}\right\}$ is sparse on $R$, then the relative harmonic dimension of $\delta R$ relative to $F=\cup K_{n}$ coincides with the harmonic dimension of $\delta R$,

$$
\operatorname{dim}_{F} \partial R=\operatorname{dim} \partial R .
$$

The proof will be given in $4.2-4.4$. 
4.2. For $W_{1}=R-K_{1}$, we have $\operatorname{dim} \delta R=\# M\left(W_{1}\right)$. Denote by $\partial X\left(\partial_{1} Y\right.$, respectively) the relative boundary of the subset $X$ (Y, respectively) of $R\left(W_{1}\right.$, respectively) relative to $R\left(W_{1}\right.$, respectively). Since $W \subset W_{1} \subset R$, we can consider both $\partial W$ and $\partial_{1} W$, with $\partial_{1} W \subsetneq \partial W$ and, in fact, $\partial W=\left(\partial_{1} W\right) \cup\left(\partial K_{1}\right)=\left(\partial_{1} W\right) \cup\left(\partial W_{1}\right)$. We consider the mapping $\lambda_{1}$ of $H P\left(W_{1}\right)$ to $H P\left(W ; \partial_{1} W\right)$ and the mapping $\mu_{1}$ of $H P\left(W ; \partial_{1} W\right)_{\mu_{1}}$ to $H P\left(W_{1}\right)$ introduced in 1.2. Here $\lambda_{1}$ defines a mapping $\lambda=\lambda_{1} \mid H P\left(W_{1} ; \partial W_{1}\right)$ of $H P\left(W_{1} ; \partial W_{1}\right)$ to $H P(W ; \partial W)$ and similarly $\mu_{1}$ defines a mapping $\mu=\mu_{1} \mid H P(W ; \partial W)_{\mu}$ of $H P(W ; \partial W)_{\mu}$ to $H P\left(W_{1} ; \partial W_{1}\right)$, where $H P(W ; \partial W)_{\mu}=$ $H P(W ; \partial W) \cap H P\left(W ; \partial_{1} W\right)_{\mu_{1}}$. The properties corresponding to (a), (b), (c), and (d) in 1.2 are readily verified to hold for the present $\lambda$ and $\mu$. We shall refer to these properties again as (a), (b), (c), and (d).

4.3. Let $u$ be minimal in $H P\left(W_{1} ; \partial W_{1}\right)$. We normalize $u$ by $\beta u(a)=1$, i.e., $\beta u \in M\left(W_{1}\right)$. Then $\beta u=k_{W_{1}}(\cdot, p)$ for some $p$ in $Q_{1}\left(W_{1}\right)$. The Brelot theorem (cf., e.g., [3], p. 139) states that any minimal point $p$ in $W_{1}^{*}-W_{1}$ is accessible from $W_{1}$ in the topology of $W_{1}^{*}$, so that for $p \in Q_{1}\left(W_{1}\right)$ there exists a curve $\gamma$ in $W_{1}$ converging to $\delta R$, and to $p$ in $W_{1}^{*}$. Since $\left\{K_{n}\right\}$ is sparse on $R$, there exists a sequence $\left\{\zeta_{n}\right\} \subset \gamma$ such that $\zeta_{n} \rightarrow \delta R$ and also $\zeta_{n} \rightarrow p$ in $W_{1}^{*}$ and $\lim _{n \rightarrow \infty} g_{W}\left(\zeta_{n}, z\right)=v(z)>0$ for every $z \in W_{1}$. We can assume, moreover, that $\lim _{n \rightarrow \infty} g_{W}\left(\zeta_{n}, z\right)$ exists for $z$ in $R$. Since $g_{W_{1}}\left(\zeta_{n}, z\right) \geqq$ $g_{W}\left(\zeta_{n}, z\right), \alpha=\lim _{n \rightarrow \infty} g_{W_{1}}\left(\zeta_{n}, a\right)>0$, and

$$
\begin{aligned}
\beta u(z) & =k_{W_{1}}(z, p)=\lim _{n \rightarrow \infty}\left(g_{W_{1}}\left(\zeta_{n}, z\right) / g_{W_{1}}\left(\zeta_{n}, a\right)\right) \\
& \geqq \frac{1}{\alpha} \lim _{n \rightarrow \infty} g_{W}\left(\zeta_{n}, z\right)=\frac{1}{\alpha} v(z)>0 .
\end{aligned}
$$

We conclude that $\lambda u \geqq(\alpha \beta)^{-1} v>0$ because $v \in H P(W ; \partial W)$, and, by (d), $\lambda u$ is minimal in $H P(W ; \partial W)$. Since $\lambda u \leqq u$ for $u \in H P\left(W_{1} ; \partial W_{1}\right)$, we have $\lambda u \in$ $H P(W ; \partial W)_{\mu}$ and $\mu \lambda u \leqq u$. There exists a positive constant $c$ with $\mu \lambda u=c u$, because $u$ is minimal in $H P\left(W_{1} ; \partial W_{1}\right)$. Thus $c \lambda u=\lambda(c u)=\lambda(\mu \lambda u)=(\lambda \mu)(\lambda u)=\lambda u$ by (a). Since $\lambda u$ is minimal and, in particular, $\lambda u>0$ on $W$, we have $c=1$. A fortiori $\mu \lambda u=u$ for minimal $u$ in $H P\left(W_{1} ; \partial W_{1}\right)$. Suppose $u_{1}$ and $u_{2}$ are minimal in $H P\left(W_{1} ; \partial W_{1}\right)$, and $\lambda u_{1}=\lambda u_{2}$. Then $u_{1}=\mu \lambda u_{1}=\mu \lambda u_{2}=u_{2}$. Therefore, we can define an injective mapping of $M\left(W_{1}\right)$ to $M(W)$ and infer that $\operatorname{dim} \delta R \leqq \operatorname{dim}_{F} \delta R$.

4.4. Conversely, let $v$ be minimal in $H P(W ; \partial W)$. We again normalize $v$ by $\beta v(a)=1$ for a reference point $a$ in $W$. Then $\beta v=k_{W}(\cdot, q)$ for some $q \in Q_{1}(W)$. Again by the Brelot theorem there exists a curve $\gamma$ in $W$ converging to $\delta R$ such that $\zeta_{n}$ in $\gamma$ converges to $\delta R$ and also to $q$ in $W^{*}$, and $\lim _{n \rightarrow \infty} g_{W}\left(\zeta_{n}, z\right)=w(z)>0$ for every $z$ in $R$; this is possible since $\left\{K_{n}\right\}$ is sparse on $R$. Hence

$$
\beta v=k_{W}(\cdot, q)=\lim _{n \rightarrow \infty} \frac{g_{W}\left(\zeta_{n}, \cdot\right)}{g_{W}\left(\zeta_{n}, a\right)}=\frac{w}{w(a)}
$$


and $w=\beta w(a) v$ is also minimal in $H P(W ; \partial W)$. We may assume, moreover, that $\lim _{n \rightarrow \infty} g_{W_{1}}\left(\zeta_{n}, z\right)=u(z)$ exists for every $z \in W_{1}$ by choosing a subsequence if necessary. Since $g_{W}\left(\zeta_{n}, z\right) \leqq g_{W_{1}}\left(\zeta_{n}, z\right)$, we obtain $w \leqq u$ on passing to the limit. Thus $w \epsilon$ $H P(W ; \partial W)_{\mu}$. The function $\mu w$ is minimal in $H P\left(W_{1} ; \partial W_{1}\right)$ by $(c)$. Since $\mu$ is injective, we can define an injective mapping of $M(W)$ to $M\left(W_{1}\right)$, and obtain $\operatorname{dim}_{F} \delta R$ $\leqq \operatorname{dim} \delta R$. In view of the result in 4.3, we have shown that $\operatorname{dim}_{F} \delta R=\operatorname{dim} \delta R$.

\section{Countably infinite harmonic dimension}

5.1. As an application of the identity theorem established in 4.1 we shall give a new proof of the following theorem originally obtained by Kuramochi [5] (see also Segawa [11]):

Theorem. There exists an open Riemann surface $R$ of Heins type such that $\operatorname{dim} \delta R=\mathfrak{a}$, the cardinal number of a countably infinite set.

The surface $R$ we are going to construct will be an infinitely sheeted unlimited covering surface of the punctured sphere $\hat{C}_{0}: 0<|z| \leqq \infty$ whose projections of branch points are all in the punctured disk $\Delta_{0}: 0<|z|<1$. From each sheet of $R$ we remove a disk $1 \leqq|z| \leqq \infty$ and obtain $F=\cup_{1}^{\infty} K_{n}$, where the $K_{n}$ are duplicates of $1 \leqq|z| \leqq \infty$ lying in each sheet of $R$. By a judicious choice of the branch points of $R$ we can see to it that $\left\{K_{n}\right\}$ is sparse on $R$, and $\operatorname{dim}_{F} \delta R=\mathfrak{a}$. Then we apply Theorem 4.1 to conclude that $\operatorname{dim} \delta R=\operatorname{dim}_{F} \delta R=\mathfrak{a}$. This is a rough sketch of the construction and reasoning we are going to develop in 5.2-5.7.

5.2. Let $\left\{a_{n}\right\}_{1}^{\infty}$ be a strictly decreasing zero sequence in $(0,1)$, and $\left\{\theta_{m}\right\}_{1}^{\infty}$ a strictly increasing sequence in $(-\pi / 2, \pi / 2)$. We then choose a decreasing zero sequence $\left\{d_{n}\right\}$ of positive numbers $d_{n}$ as follows. Let $D_{n m}=\left\{\left|z-a_{n} e^{i \theta_{m}}\right| \leqq d_{n}\right\}$. We make $\left\{d_{n}\right\}$ converge to zero so rapidly that any two closed disks in the family $\bigcup_{m=1}^{\infty}\left\{D_{n m} ; n \geqq m\right\}$ are disjoint. We set

$$
\left\{\begin{array}{l}
D(m)=\bigcup_{n \geqq m} D_{n m} \quad(m=1,2, \ldots), \\
D(0)=\bigcup_{m=1}^{\infty} D(m)=\bigcup_{m=1}^{\infty}\left(\bigcup_{n \geqq m} D_{n m}\right) .
\end{array}\right.
$$

We fix sequences $\left\{a_{n}\right\},\left\{\theta_{m}\right\}$, and an auxiliary sequence $\left\{d_{n}\right\}$ once and for all. We then choose a strictly decreasing zero sequence $\left\{b_{n}\right\}_{1}^{\infty}$ in $(0,1)$ such that $a_{n+1}<b_{n}<a_{n}$ $(n=1,2, \ldots)$. Let $I_{n m}=\left\{b_{n} \leqq|z| \leqq a_{n}\right.$, arg $\left.z=\theta_{m}\right\}$, a radial line segment. First of all we require that each $I_{n m}$ is contained in the interior of $D_{n m}(n \geqq m)$. Set

$$
\left\{\begin{array}{l}
I(m)=\bigcup_{n \geqq m} I_{n m} \quad(m=1,2, \ldots), \\
I(0)=\bigcup_{m=1}^{\infty} I(m)=\bigcup_{m=1}^{\infty}\left(\bigcup_{n \geqq m} I_{n m}\right) .
\end{array}\right.
$$


Actually we will choose each $b_{n}$ so close to $a_{n}$ that it satisfies not only the above requirement but also the conditions $(\mathrm{A})$ and $(\mathrm{B})$ to be specified later.

5.3. Using a countably infinite number of duplicates of the punctured sphere $\hat{C}_{0}: 0<|z| \leqq \infty$, and the slits $I(0)$ and $I(m)$, we form the disjoint sheets

$$
R_{m}=\hat{C}_{0}-I(m) \quad(m=0,1, \ldots) .
$$

Then we join each $R_{m}(m=1,2, \ldots)$ to $R_{0}$ crosswise along the slits $I(m)$ and denote the resulting surface by $R$. It is a covering surface of $\hat{C}_{0}$ with the natural projection mapping $\pi$. It is not difficult to see that $R$ is an open Riemann surface of Heins type.

In each $R_{m}(m=0,1, \ldots)$, take the closed disk $K_{m}=\{1 \leqq|z| \leqq \infty\}$. Clearly $\left\{K_{m}\right\}_{0}^{\infty}$ is a $\mathscr{K}$-sequence on $R$. We set

$$
F=\bigcup_{0}^{\infty} K_{m}, \quad W=R-F,
$$

and

$$
W_{m}=R_{m}-K_{m} \quad(m=0,1, \ldots) .
$$

Then $W$ is also obtained by joining $W_{m}$ to $W_{0}$ crosswise along the slits $I(m)$ $(m=1,2, \ldots)$. Thus it is a covering surface of the punctured disk $\Delta_{0}: 0<|z|<1$ with the natural projection $\pi$.

5.4. Fix a number $c$ in $\left(a_{1}+d_{1}, 1\right)$. The circle $C_{m}=\{|z|=c\}$ is contained in $W_{m}$ and so is the annulus $\{c<|z|<1\}(m=0,1, \ldots)$. Let $w$ be the harmonic function on $\{0<|z|<c\}-I(0)$ with boundary values 1 on $|z|=c$ and 0 on $I(0)$. We now choose each $b_{n}$ so close to $a_{n}$ that the following condition is satisfied:

$$
\eta_{A}^{\prime}=\inf \{w(z) ; z \in\{0<|z|<c\}-D(0)\}>0 .
$$

As a consequence of this choice of $\left\{b_{n}\right\}_{1}^{\infty}$, the $\mathscr{K}$-sequence $\left\{K_{m}\right\}_{0}^{\infty}$ in $R$ is sparse on $R$.

To prove this we set

$$
G_{m}=\{0<|z|<c\}-I(m) \subset W_{m} \subset R_{m} \quad(m=0,1, \ldots) .
$$

Then $w$ can be considered subharmonic on each $G_{m}(m=0,1, \ldots)$ by defining $w=0$ on $I(0)$. Let $\gamma$ be an arbitrary curve in $R$ tending to $\delta R$, and denote by $g_{W}(\cdot, \zeta)$ the Green's function on $W$ with pole $\zeta$ in $W$ and extended as zero to $R-W$. We are to show that

$$
\limsup _{z \in \gamma, z \rightarrow \delta R} g_{W}(z, \zeta)>0
$$

for one and hence for every $\zeta$ in $W$. Observe that $\pi(\gamma)$ is a curve in $\hat{\boldsymbol{C}}_{\mathbf{0}}$ tending to the origin 0 .

First we consider the case in which there exists a single $G_{m}$ such that $\gamma \subset G_{m}$. Choose a sequence $\left\{z_{n}\right\}$ in $\gamma \cap\left(G_{m}-D(m)\right)$ such that $z_{n} \rightarrow \delta R$. Let $\alpha=\inf _{C_{m}} g_{W}(\cdot, \zeta)$ $>0$. Then clearly $g_{W}(\cdot, \zeta) \geqq \alpha w$ on $G_{m}$. In view of $(\mathrm{A})$ we have

$$
\limsup _{z \in \gamma, z \rightarrow \delta R} g_{W}(z, \zeta) \geqq \limsup _{n \rightarrow \infty} g_{W}\left(z_{n}, \zeta\right) \geqq \alpha \limsup _{n \rightarrow \infty} w\left(z_{n}\right) \geqq \alpha \eta_{A}>0 \text {. }
$$


Next we consider the case in which the above alternative does not occur, so that there exists a sequence $\left\{z_{n}\right\}$ in $\gamma \cap\left(G_{m(n)}-D(m(n))\right.$ such that $z_{n} \rightarrow \delta R$ and $m(n) \neq$ $m\left(n^{\prime}\right)\left(n \neq n^{\prime}\right)$. Let $\gamma_{n}$ be that part of $\gamma$ which starts from $z_{n}$ and ends at $z_{n+1}$. In view of the construction of $R, \gamma_{n}$ must pass through $G_{0}-D(0)$ and, therefore, we can choose a point $w_{n}$ in $\gamma_{n} \cap\left(G_{0}-D(0)\right)$. Then $w_{n} \rightarrow \delta R$, and in the same fashion as above we conclude that

$$
\limsup _{z \in \gamma, z \rightarrow \delta R} g_{W}(z, \zeta)>0 .
$$

5.5. It is readily seen that there exists on $0<|z| \leqq 1$ a unique smallest function $l$ in the family of continuous functions $v$ on $0<|z| \leqq 1$ which are harmonic on $\{0<|z|<1\}-I(0)$ and satisfy $v(z)=0$ on $|z|=1$, and $v(z)=\log (2 /|z|)$ on $I(0)$. We now impose upon the closeness of $b_{n}$ to $a_{n}$ the additional condition

$$
\eta_{B}=\sup \{l(z) ; z \in(-1,0)\}<+\infty .
$$

The function $l$ may be viewed as being defined and superharmonic on each $W_{m}$ $(m=0,1, \ldots)$.

Denote by $L_{m}$ the segment $(-1,0)$ in $W_{m}(m=0,1, \ldots)$. Fix an $m$ for the time being and choose a sequence $\left\{-t_{n}\right\}_{1}^{\infty} \subset L_{m}$ such that $-t_{n} \rightarrow 0$ and $g_{W}\left(z,-t_{n}\right)$ is convergent for each $z$ in $W$. On setting $\alpha=\inf _{C_{m}} g_{W}(z, \cdot)$, we see that $g_{W}\left(z,-t_{n}\right) \geqq$ $\alpha w\left(-t_{n}\right) \geqq \alpha \eta_{A}>0$. Therefore,

$$
u_{m}(z)=\lim _{n \rightarrow \infty} g_{W}\left(z,-t_{n}\right)>0
$$

for $z \in W$, and $u_{m} \in H P(W ; \partial W)$.

We now study the growth of $u_{m}$. Let

$$
h_{\zeta}(z)=\log \left|\frac{1-\bar{\zeta} z}{z-\zeta}\right| \quad(|z|,|\zeta|<1) .
$$

We can lift $h .(\cdot)$ to $W \times W$ from $\Delta_{0} \times \Delta_{0}$ by

$$
h_{\zeta}(z)=h_{\pi(\zeta)}(\pi(z)) \quad((z, \gamma) \in W \times W) .
$$

The discussion in what follows will be based on the inequality

$$
g_{W}(\cdot, \zeta) \leqq h_{\zeta} \quad\left(=h_{\pi(\zeta)} \circ \pi\right)
$$

on $W$ for any $\zeta$ in $W$. If $z \in I\left(m^{\prime}\right)\left(m^{\prime}=0,1, \ldots\right)$, then $\operatorname{Re} z>0$ and

By the maximum principle,

$$
g_{W}\left(z,-t_{n}\right) \leqq h_{-t_{n}}(z)=\log \left|\frac{1+t_{n} z}{z+t_{n}}\right| \leqq \log \frac{2}{|z|}=l(z) .
$$

$$
g_{W}\left(z,-t_{n}\right) \leqq l(z) \quad\left(z \in W_{m^{\prime}}, m^{\prime} \neq m\right) .
$$

Observe that $h_{-t_{n}}(z)-l(z) \leqq 0$ on $I(m)$. Therefore,

$$
g_{W}\left(z,-t_{n}\right) \geqq h_{-t_{n}}(z)-l(z) \quad\left(z \in W_{m}\right),
$$


since the same is true of the boundary values on $\partial W_{m}$. On passing to the limit we conclude that

$$
\begin{gathered}
u_{m}(z) \leqq l(z) \quad\left(z \in W_{m^{\prime}}, m^{\prime} \neq m\right), \\
u_{m}(z) \geqq h_{0}(z)-l(z) \quad\left(z \in W_{m}\right) .
\end{gathered}
$$

Here $h_{0}(z)=\log (1 /|z|)$. Therefore, by (B), we have

$$
\begin{gathered}
\sup _{L_{m^{\prime}}} u_{m}<+\infty \quad\left(m^{\prime} \neq m\right), \\
\sup _{L_{m}} u_{m}=+\infty .
\end{gathered}
$$

With each $m$ we associate a $u_{m}$ as above. By using the Martin representation (7) for each function in $\left\{u_{m}\right\}_{1}^{\infty}$, we can easily see from (13) that $\# M(W) \geqq \mathfrak{a}$, i.e., $\operatorname{dim}_{\boldsymbol{F}} \delta R \geqq \mathfrak{a}$.

5.6. Take an arbitrary $u$ in $M(W)$, so that $u=k_{W}(\cdot, q)$ for some $q \in Q_{1}(W)$. By the Brelot theorem there exists a curve $\gamma$ in $W$ tending to $\delta R$ and to $q$ in $W^{*}$. Since $\left\{K_{n}\right\}$ is sparse on $R$, there exists a sequence $\left\{\zeta_{n}\right\}$ in $\gamma$ tending to $\delta R$ such that $\lim _{n \rightarrow \infty} g_{W}\left(\zeta_{n}, z\right)$ exists and is positive for any $z \in W$. In view of

we set

$$
\lim _{n \rightarrow \infty} \frac{g_{W}\left(\zeta_{n}, z\right)}{g_{W}\left(\zeta_{n}, a\right)}=\lim _{n \rightarrow \infty} k_{W}\left(z, \zeta_{n}\right)=k(z, q)=u(z)
$$

$$
\beta=\lim _{n \rightarrow \infty} g_{W}\left(\zeta_{n}, a\right)>0,
$$

and obtain $\lim _{n \rightarrow \infty} g_{W}\left(z, \zeta_{n}\right)=\beta u(z)$ for any $z \in W$. By (12), $\beta u \leqq h_{0}$ on $W$. Set

$$
\beta_{u}=\sup \left\{\beta ; \beta u \leqq h_{0} \text { on } W\right\} .
$$

We have obtained a mapping $u \rightarrow \beta_{u} u=v$ from $M(W)$ onto $M^{\prime}(W)=\left\{\beta_{u} u ; u \in M(W)\right\}$, which is bijective. Thus $\# M(W)=\# M^{\prime}(W)$.

Set

$$
M_{k}^{\prime}(W)=\left\{v \in M(W) ; v(a) \geqq \frac{1}{k} h_{0}(a)\right\} \quad(k=1,2, \ldots) .
$$

Take different elements $v_{1}, \ldots, v_{n}$ in $M_{k}^{\prime}(W)$. By the Kjellberg lemma (cf., e. g., [3], p. 18), the relations $v_{j} \leqq h_{0}(j=1, \ldots, n)$ imply that $v_{1}+\ldots+v_{n} \leqq h_{0}$. Considering this at $a$ we see that

$$
\frac{n}{k} h_{0}(a) \leqq v_{1}(a)+\ldots+v_{n}(a) \leqq h_{0}(a),
$$

or $n / k \leqq 1$. Therefore, $n \leqq k$ and $\# M_{k}^{\prime}(W) \leqq k$. Since $M^{\prime}(W)=\bigcup_{k=1}^{\infty} M_{k}^{\prime}(W)$, we obtain \# $M^{\prime}(W) \leqq \mathfrak{a}$, i.e.; $\operatorname{dim}_{F} \delta R \leqq \mathfrak{a}$.

5.7. From 5.5 and 5.6 it follows that $\operatorname{dim}_{F} \delta R=\mathfrak{a}$. Since $\left\{K_{n}\right\}$ is sparse on $R$, Theorem 4.1 implies that $\operatorname{dim} \delta R=\operatorname{dim}_{F} \delta R=\mathfrak{a}$. The proof of Theorem 5.1 is complete. 


\section{References}

[1] Ancona, A.: Une propriété de la compactification de Martin d'un domaine Euclidien. - Ann. Inst. Fourier (Grenoble), 29, 1979, 71-90.

[2] Constantinescu, C., and A. Cornea: Über einige Probleme von M. Heins. - Rev. Roumaine Math. Pures Appl. 4, 1959, 277-281.

[3] Constantinescu, C., and A. Cornea: Ideale Ränder Riemannscher Flächen. - Ergebnisse der Mathematik und ihrer Grenzgebiete 32. Springer-Verlag, Berlin-Göttingen-Heidelberg, 1963.

[4] HeIns, M.: Riemann surfaces of infinite genus. - Ann. of Math. 55, 1952, 296-317.

[5] KURAMOCHI, Z.: An example of a null-boundary Riemann surface. - Osaka J. Math. 6, 1954, 83-91.

[6] NAKaI, M.: Relative harmonic dimensions. - Seminar Note at Research Inst. for Math. Sci. 366, Kyoto University, Kyoto, 1979, 137-149 (Japanese).

[7] NaKaI, M.: The range of Picard dimensions. - Proc. Japan Acad. Ser. A. Math. Sci. 55, 1979, 379-383.

[8] NAKAI, M., and L. SARIO: The range of relative harmonic dimensions. - Topology - Calculus of variations and their applications (Euler volume), Dekker (to appear).

[9] NAKaI, M., and T. TADA: The distribution of Picard dimensions. - Kodai Math. J. 7, 1984, $1-15$.

[10] Noshiro, K.: Cluster sets. - Ergebnisse der Mathematik und ihrer Grenzgebiete 28. SpringerVerlag, Berlin-Göttingen-Heidelberg, 1960.

[11] Segawa, S.: A duality relation for harmonic dimensions and its applications. - Kodai Math. J. 4, 1981, 508-514.

Nagoya Institute of Technology

Department of Mathematics

Gokiso, Showa, Nagoya 466

Japan

Received 4 January 1984
University of California

Department of Mathematics

Los Angeles, California 90024

USA 